\title{
Input-output decoupling of Hamiltonian systems: The linear case
}

\section{H. NIJMEIJER and A.J. van der SCHAFT \\ Dept. of Applied Mathematics, Twente University of Technology, P.O. Box 217, 7500 AE Enschede, The Netherlands}

Received S June 1984

Abstract: In this note we give necessary and sufficient conditions for a linear Hamiltonian system to be input-output decouplable by Hamiltonian feedback, i.e. feedback that preserves the Hamiltonian structure. In a second paper we treat the same problem for nonlinear Hamiltonian systems.

AMS Subject Classification: 93C05, 93B10, 58F05.

Keywords: Noninteracting control, Linear Hamiltonian systems, Output feedback, Symplectic structure.

\section{Introduction}

A physically well-motivated subclass of the set of all linear systems

$\dot{x}=A x+B u$,

$y=C x$,

consists of the Hamiltonian systems. Such a Hamiltonian system is defined as follows. The state space $X$ is even dimensional, say $X=\mathbb{R}^{2 n}$ and is endowed with a symplectic form

$J=\left(\begin{array}{cc}0 & -I_{n} \\ I_{n} & 0\end{array}\right)$.

Furthermore the matrices $A, B$ and $C$ satisfy

$A^{\mathrm{T}} J+J A=0 \quad$ ( $A$ is Hamiltonian),

$B_{j}^{\mathrm{T}}=C$.

It follows that necessarily the number of inputs equals the number of outputs, say $u, y \in \mathbb{R}^{m}$. Without loss of generality we may assume that $B$ is injective, or equivalently, $C$ is surjective. From an input-output point of view a Hamiltonian system is fully characterized by the condition $G(t)=$ $-G^{\mathrm{T}}(-t)$, or $H(s)=H^{\mathrm{T}}(-s)$, where $G$ resp. $H$ is the impulse response resp. transfer matrix of (1.1), see e.g. $[1,7,8]$. Many physical systems are of this Hamiltonian form, for example linear conservative mechanical systems. In a second paper [11] we shall consider nonlinear Hamiltonian systems (cf. [8]), but we mention already that the linearization of a nonlinear Hamiltonian system (e.g. a robot manipulator) also exhibits this linear Hamiltonian structure (1.2).

In this paper we wish to tackle the input-output decoupling problem for linear Hamiltonian systems; that is we seek for a feedback $u=F x+G v$ for the Hamiltonian systems (1.1), such that in the modified system

$\dot{x}=(A+B F) x+B G v$,

$y=C x$,

with $v=\left(v_{1}, \ldots, v_{m}\right)^{\mathrm{T}}, y=\left(y_{1}, \ldots, y_{m}\right)^{\mathrm{T}}$, the input $v_{i}$ does not affect the output $y_{j}$ for $j \neq i$. Moreover we require the input $v_{i}$ to 'control' the output $y_{i}$, $i=1, \ldots, m$. Necessary and sufficient conditions for the solvability of this problem are well known, cf. $[2,5]$. However in this paper we shall add the extra requirement that the system (1.3) after feedback is again Hamiltonian. This restricts the allowed feedback in the following way.

Proposition 1.1. Suppose (1.1) defines a Hamiltonian system. The system (1.3) is again Hamiltonian if and only if

(a) $G=I_{m}$,

(b) $F=H C$ for some $(m, m)$-matrix $H$ with $H=H^{\mathrm{T}}$.

Proof (see [7,8]). The triple $(A+B F, B G, C)$ has to satisfy (1.2), i.e.

$(A+B F)^{\mathrm{T}} J+J(A+B F)=0, \quad(B G)^{\mathrm{T}} J=C$.

From the last identity we obtain $G=I_{m}$. The first equation yields

$F^{\mathrm{T}} B^{\mathrm{T}} J+J B F=F^{\mathrm{T}} C-C^{\mathrm{T}} F=0$. 
Therefore the symplectic form

$\left(\begin{array}{cc}0 & -I_{m} \\ I_{m} & 0\end{array}\right)$

on $\mathbb{R}^{2 m}$ is zero restricted to the subspace $\operatorname{Im}\left({ }_{F}^{C}\right)$, and this implies that

$\operatorname{dim} \operatorname{Im}\left(\begin{array}{l}C \\ F\end{array}\right) \leqslant m$.

Since rank $C=m$ it follows $F=H C$ for some $(m, m)$-matrix $H$. Now from $F^{\mathrm{T}} C-C F^{\mathrm{T}}=0$ this yields $C^{\mathrm{T}} H^{\mathrm{T}} C-C^{\mathrm{T}} H C=0$ or $H=H^{\mathrm{T}}$.

Such a feedback $u=H C x+v=H y+v$, with $H=H^{\mathrm{T}}$, which leaves the Hamiltonian structure invariant, will be called a Hamiltonian feedback. From a physical viewpoint such a feedback adds a 'potential energy' $\frac{1}{2} y^{\mathrm{T}} H y$ to the internal energy of the system, while leaving the input channels invariant (i.e. no precompensation factor $G$ ). Now the Hamiltonian input-output decoupling problem can be formulated as follows: Consider the Hamiltonian system (1.1). Find, if possible, an output feedback $u=H y$, with $H=H^{\mathrm{T}}$, such that in

$\dot{x}=(A+B H C) x+B u$,

$y=C x$,

the transfer functions from $u_{i}$ to $y_{j}$ are zero for $j \neq i$ and nonzero for $i=j, i, j=1, \ldots, m$.

\section{Geometric and Hamiltonian preliminaries}

Recall the following notions from the geometric approach to linear system theory, cf. $[9,10]$. A subspace $V \subset X$ is called $(A, B)$-invariant for the - not necessarily Hamiltonian - system (1.1) if $A V \subset V+\operatorname{Im} B$,

or equivalently, if there exists a matrix $F$ such that $(A+B F) V \subset V$. The maximal $(A, B)$-invariant subspace in $\operatorname{Ker} C$ (which exists) is denoted by $V^{*}(A, B, C)$. A subspace $R \subset X$ is called a controllability subspace of (1.1) if $R$ is $(A, B)$-invariant and the eigenvalues of $A+B F \mid R$ can arbitrarily be assigned by using an appropriate feedback $F$ which leaves $R$ invariant: $(A+B F) R$ $\subset R$. The maximal controllability subspace contained in $\operatorname{Ker} C$ (which also exists) is denoted by $R^{*}(A, B, C)$. Furthermore a subspace $S \subset X$ is called $(C, A)$-invariant if

$A(S \cap \operatorname{Ker} C) \subset S$,

or equivalently, if there exists a matrix $K$ such that $(A+K C) S \subset S$. The minimal $(C, A)$-invariant subspace containing $\operatorname{Im} B$ (which exists) will be denoted as $S^{*}(A, B, C)$. We will now derive some elementary facts on $(A, B)$ - and $(C, A)$-invariant subspaces of a Hamiltonian system, see also [7]. Therefore assume that the state space $X$ equals $\mathbb{R}^{2 n}$ and is endowed with a symplectic form $J$. For a linear subspace $V \subset X$ the orthogonal complement of $V$ with respect to $J$, denoted as $V^{\perp}$, is given by

$V^{\perp}=\left\{x \in \mathbb{R}^{2 n} \mid x^{\mathrm{T}} J v=0, \forall v \in V\right\}$.

Proposition 2.1. Let $(A, B, C)$ be a Hamiltonian system. Then

(a) $(A, B)$ is controllable $\Leftrightarrow(C, A)$ is observable.

(b) $V \subset \mathbb{R}^{2 n}$ is $(A, B)$-invariant if and only if $V^{\perp}$ is $(C, A)$ invariant.

(c) $V^{*}(A, B, C)=\left(S^{*}(A, B, C)\right)^{\perp}$.

(d) $R^{*}(A, B, C)=V^{*}(A, B, C) \cap$

$\left(V^{*}(A, B, C)\right)^{\perp}$.

Proof. (a) Check the rank conditions for controllability and observability by using (1.2).

(b) Suppose $V \subset \mathbb{R}^{2 n}$ satisfies

$A V \subset V+\operatorname{Im} . B$.

Then

$V^{\perp} \cap(\operatorname{Im} B)^{\perp} \subset(A V)^{\perp}$.

Now

$$
\begin{aligned}
x \in(A V)^{\perp} & \Leftrightarrow x^{\mathrm{T}} J A v=0, \forall v \in V \\
& \Leftrightarrow x^{\mathrm{T}} A^{\mathrm{T}} J v=0, \forall v \in V \\
& \Leftrightarrow A x \in V^{\perp} .
\end{aligned}
$$

Hence $(A V)^{\perp}=A^{-1}\left(V^{\perp}\right)$ (here $A^{-1} W:=\{x \in$ $\left.\mathbb{R}^{2 n} \mid A x \in W\right\}$ for a subspace $\left.W\right)$. Furthermore, since $B^{\mathrm{T}} J=C$ we have $(\operatorname{Im} B)^{\perp}=\operatorname{Ker} C$. So

$V^{\perp} \cap \operatorname{Ker} C \subset A^{-1}\left(V^{\perp}\right)$

or $A\left(V^{\perp} \cap \operatorname{Ker} C\right) \subset V^{\perp}$.

(c) Follows directly from (b). (In fact it is easily seen that the subspaces $V^{i}$ and $S^{i}$ in the $V^{*}$ and $S^{*}$ algorithm, cf. $[9,10]$, are dual in the sense that $V^{i}=\left(S^{i}\right)^{\perp}$.) 
(d) Use the fact that

$R^{*}(A, B, C)=V^{*}(A, B, C) \cap S^{*}(A, B, C)$,

cf. [4] and (c).

\section{Main theorem}

Let $(A, B, C)$ be a Hamiltonian system on $\left(\mathbb{R}^{2 n}, J\right)$. A subspace $V \subset \mathbb{R}^{2 n}$ is called symplectic if $V^{\perp} \cap V=0$. We denote by $b_{i}$ the $i$-th column of the matrix $B$ and by $c_{i}$ the $i$-th row of the matrix $C, i=1, \ldots, m$. Furthermore $\hat{B}_{i}$ is the $(2 n, m-1)$ matrix derived from $B$ by deleting the $i$-th column $b_{i}, i=1, \ldots, m$. Similarly $\hat{C}_{i}$ is the $(m-1,2 n)$ matrix resulting from $C$ by deleting $c_{i}, i=1, \ldots, m$. Our main theorem is now as follows.

Theorem 3.1. Let $(A, B, C)$ be a minimal Hamiltonian system with $V^{*}(A, B, C)=0$. Then the Hamiltonian input-output decoupling problem can be solved if and only if the following two conditions are satisfied:

(a) $b_{i} \in V^{*}\left(A, B, \hat{C}_{i}\right), i=1, \ldots, m$,

(b) $V^{*}\left(A, B, C_{i}\right)=\left(V^{*}\left(A, B, \hat{C}_{i}\right)\right)^{\perp}, \quad i=$ $1, \ldots, m$.

Proof. For simplicity we will write

$V_{i}^{*}=V^{*}\left(A, B, \hat{C}_{i}\right)$ and $\hat{V}_{i}^{*}=V^{*}\left(A, B, c_{i}\right)$.

(If) Since $b_{i} \in V^{*}$ it follows that

$V_{i}^{*}=V^{*}\left(A, B, \hat{C}_{i}\right)=V^{*}\left(A, \hat{B}_{i}, \hat{C}_{i}\right)$

and, since $\hat{V}_{i}^{*}=\sum_{j \neq i} V_{j}^{*}$, that

$\hat{V}_{i}^{*}=V^{*}\left(A, B, c_{i}\right)=V^{*}\left(A, b_{i}, c_{i}\right)$.

Now the triple $\left(A, b_{i}, c_{i}\right)$ is again a Hamiltonian system (with scalar input and output) and therefore we can apply Proposition 2.1 to conclude that

$R^{*}\left(A, b_{i}, c_{i}\right)=\hat{V}_{i}^{*} \cap\left(\hat{V}_{i}^{*}\right)^{\perp}$.

Now suppose that

$\hat{R}_{i}^{*}:=R^{*}\left(A, b_{i}, c_{i}\right) \neq 0$.

Then we have $b_{i} \in \hat{R}_{i}^{*}$ and hence

$A \hat{R}_{i}^{*} \subset \hat{R}_{i}^{*}+b_{i}=\hat{R}_{i}^{*}$

and also by definition $\hat{R}_{i}^{*} \subset \operatorname{Ker} c_{i}$. Furthermore $\hat{R}_{i}^{*} \subset\left(\hat{V}_{i}^{*}\right)^{\perp}=V_{i}^{*}$ and so $\hat{R}_{i}^{*} \subset \operatorname{Ker} \hat{C}_{i}$. Therefore
$\hat{R}_{i}^{*}$ is $A$-invariant and is contained in $\operatorname{Ker} C$. Hence if $\hat{R}_{i}^{*} \neq 0$ we obtain a contradiction with the observability of $(A, B, C)$. It follows that $\hat{V}_{i}^{*}$ $\cap\left(\hat{V}_{i}^{*}\right)^{\perp}=0$ and hence $\hat{V}_{i}^{*}$ is a symplectic subspace of $\left(\mathbb{R}^{2 n}, J\right)$ for all $i=1, \ldots, m$. Since $V_{i}^{*}=$ $\left(\hat{V}_{i}^{*}\right)^{\perp}$ this also yields that $V_{i}^{*}$ is symplectic for all $i=1, \ldots, m$. Moreover

$$
0=\hat{V}_{i}^{*} \cap\left(\hat{V}_{i}^{*}\right)^{\perp}=\hat{V}_{i}^{*} \cap V_{i}^{*}=\sum_{j \neq i} V_{j}^{*} \cap V_{i}^{*}
$$

implies that the subspaces $V_{1}^{*}, \ldots, V_{m}^{*}$ are independent (cf. [10]). This yields that there exists an $(m, 2 n)$-matrix $F$ such that

$$
(A+B F) V_{i}^{*} \subset V_{i}^{*}, \quad i=1, \ldots, m
$$

(this also follows from the "noninteracting condition' $\left.b_{i} \in V_{i}^{*}\right)$. Moreover

$\operatorname{Im} B \subset \sum_{i=1}^{m} V_{i}^{*}, \quad A\left(\sum_{i=1}^{m} V_{i}^{*}\right) \subset \sum_{i=1}^{m} V_{i}^{*}$

and hence by controllability $\mathbb{R}^{2 n}=V_{1}^{*} \oplus \cdots \oplus V_{m}^{*}$. Furthermore because $V_{i}^{*}=\left(\hat{V}_{i}^{*}\right)^{\perp}$ and $\hat{V}_{i}^{*}$ is $(A, B)$-invariant we obtain by Proposition 2.1 that $V_{i}^{*}$ is also $(C, A)$-invariant, $i=1, \ldots, m$. Therefore there exists an $(m, m)$-matrix $H$ such that

$$
(A+B H C) V_{i}^{*} \subset V_{i}^{*}, \quad i=1, \ldots, m .
$$

The last thing we have to show is that we may take $H$ to be a symmetric matrix.

Since $V_{i}^{*}$ is symplectic there exists a symplectic basis $\left(q_{i}, p_{i}\right)$ for $V_{i}^{*}$, i.e. a basis

$\left(q_{i}, p_{i}\right)=\left(q_{i}^{1}, \ldots, q_{i}^{k_{i}}, p_{i}^{1}, \ldots, p_{i}^{k_{i}}\right)$

with $\operatorname{dim} V_{i}^{*}=2 k_{i}$, such that $J$ restricted to $V_{i}^{*}$ equals

$\left(\begin{array}{cc}0 & -I_{k_{i}} \\ I_{k_{i}} & 0\end{array}\right)$

in this basis. Then because $\left(V_{i}^{*}\right)^{\perp}=\sum_{j \neq i} V_{j}^{*}$ it follows that $\left(q_{1}, \ldots, q_{n}, q_{1}, \ldots, p_{n}\right)$ is a symplectic basis for $\mathbb{R}^{2 n}$, with

$J=\left(\begin{array}{cc}0 & -I_{n} \\ I_{n} & 0\end{array}\right)$

Next we will use the relations

$(A+B H C) V_{i}^{*} \subset V_{i}^{*}, \quad i=1, \ldots, m$. 
We have

$$
\begin{array}{ll}
(A+B H C) V_{1}^{*} \subset V_{1}^{*}, & V_{1}^{*} \subset \operatorname{Ker} \hat{C}_{1}, \\
(A+B H C) \hat{V}_{1}^{*} \subset \hat{V}_{1}^{*}, & \hat{V}_{1}^{*} \subset \operatorname{Ker} c_{1} .
\end{array}
$$

Hence

$$
\begin{aligned}
(J A+J B H C) V_{1}^{*} & =\left(-Q-C^{\top} H C\right) V_{1}^{*} \\
& \subset J V_{1}^{*}=V_{1}^{*}
\end{aligned}
$$

and

$$
\left(-Q-C^{\mathrm{T}} H C\right) \hat{V}_{1}^{*}=\hat{V}_{1}^{*},
$$

where $Q=-J A$ is symmetric. Writing out in the above basis we get

$$
\begin{aligned}
& \left(Q+C^{\mathrm{\top}} H C\right)\left(\begin{array}{l}
* \\
0 \\
* \\
0
\end{array}\right) \subset\left(\begin{array}{l}
* \\
0 \\
* \\
0
\end{array}\right), \\
& \left(Q+C^{\top} H C\right)\left(\begin{array}{l}
0 \\
* \\
0 \\
*
\end{array}\right) \subset\left(\begin{array}{l}
0 \\
* \\
0 \\
*
\end{array}\right),
\end{aligned}
$$

where we have symbolically written

$$
V_{1}^{*}=\left(\begin{array}{c}
* \\
0 \\
* \\
0
\end{array}\right), \quad \hat{V}_{1}^{*}=\left(\begin{array}{c}
0 \\
* \\
0 \\
*
\end{array}\right) .
$$

In the same way the matrix $C$ can be expressed in this basis as

$$
C=\left(\begin{array}{c}
c_{1} \\
\hat{C}_{1}
\end{array}\right)=\left(\begin{array}{cccc}
c_{11} & 0 & c_{13} & 0 \\
0 & C_{22} & 0 & C_{24}
\end{array}\right) \text {. }
$$

Furthermore the matrices $Q$ and $H$ will be partitioned according to this basis and we obtain that $Q$ and $C^{\mathrm{T}} H C$ have the following form respectively:

$$
\begin{aligned}
& \left(\begin{array}{llll}
Q_{11} & Q_{12} & Q_{13} & Q_{14} \\
Q_{21} & Q_{22} & Q_{23} & Q_{24} \\
Q_{31} & Q_{32} & Q_{33} & Q_{34} \\
Q_{41} & Q_{42} & Q_{43} & Q_{44}
\end{array}\right), \\
& \left(\begin{array}{llll}
c_{11}^{\mathrm{T}} h_{11} c_{11} & c_{11}^{\mathrm{T}} h_{12} C_{22} & c_{11}^{\mathrm{T}} h_{11} c_{13} & c_{11}^{\mathrm{T}} h_{12} C_{24} \\
C_{22}^{\mathrm{T}} h_{21} c_{11} & C_{22}^{\mathrm{T}} H_{22} C_{22} & C_{22}^{\mathrm{T}} h_{21} c_{13} & C_{22}^{\mathrm{T}} H_{22} C_{24} \\
c_{13}^{\mathrm{T}} h_{11} c_{11} & c_{13}^{\mathrm{T}} h_{12} C_{22} & c_{13}^{\mathrm{T}} h_{11} c_{13} & c_{13}^{\mathrm{T}} h_{12} C_{24} \\
C_{24}^{\mathrm{T}} h_{21} c_{11} & C_{24}^{\mathrm{T}} H_{22} C_{22} & C_{24}^{\mathrm{T}} h_{21} c_{13} & C_{24}^{\mathrm{T}} H_{22} C_{24}
\end{array}\right),
\end{aligned}
$$

and so the matrix $Q+C^{\mathrm{T}} H C$ leaves the subspaces

$$
\left(\begin{array}{l}
* \\
0 \\
* \\
0
\end{array}\right) \text { and }\left(\begin{array}{l}
0 \\
* \\
0 \\
*
\end{array}\right)
$$

invariant. This yields

$$
\begin{aligned}
& c_{11}^{\mathrm{T}} h_{12} C_{22}+Q_{12}=0, \\
& C_{22}^{\mathrm{T}} h_{21} c_{11}+Q_{21}=0, \\
& C_{22}^{\mathrm{T}} h_{21} c_{13}+Q_{23}=0, \\
& c_{13}^{\mathrm{T}} h_{12} C_{22}+Q_{32}=0, \\
& c_{11}^{\mathrm{T}} h_{12} C_{24}+Q_{14}=0, \\
& C_{24}^{\mathrm{T}} h_{21} c_{11}+Q_{41}=0, \\
& c_{13}^{\mathrm{T}} h_{12} C_{24}+Q_{34}=0, \\
& C_{24}^{\mathrm{T}} h_{21} c_{13}+Q_{43}=0 .
\end{aligned}
$$

Now the matrix $Q$ is symmetric, so $q_{12}=q_{21}^{\top}$, $q_{23}=q_{32}^{\top}, q_{14}=q_{41}^{\top}$ and $q_{34}=q_{43}^{\top}$. It follows that we may take $h_{21}=h_{12}^{\mathrm{T}}$ in (3.1). Having fixed $h_{11}$ and $h_{12}=h_{21}^{\mathrm{T}}$ we proceed by considering $V_{2}^{*}$ and $\hat{V}_{2}^{*}$ (or $\hat{V}_{2}^{*} \backslash V_{1}^{*}$ ). Continuing up to $V_{m}^{*}$ and $\hat{V}_{m}^{*}$ in this way we conclude that we may take $H$ satisfying

$(A+B H C) V_{i}^{*} \subset V_{i}^{*}, \quad i=1, \ldots, m$,

to be symmetric. Therefore it follows from $b_{i} \in V_{i}^{*}$, $i=1, \ldots, m$, that $u=H y+v$ is a Hamiltonian feedback which decouples the system. Clearly the subsystems

$\left(A+B H C \mid V_{i}^{*}, b_{i}, c_{i}\right), \quad i=1, \ldots, m$,

are minimal Hamiltonian systems.

(Only if) It is well known that the input-output decoupling by general state feedback is possible if and only if

$\operatorname{Im} B=\sum_{i=1}^{m} \operatorname{Im} B \cap V_{i}^{*}$,

see [5]. Since the precompensation matrix $G$ equals $I_{m}$, it follows that $b_{i} \in V_{i}^{*}, i=1, \ldots, m$. Because $V^{*}(A, B, C)=0$ we must have

$(A+B H C) V_{i}^{*} \subset V_{i}^{*}, \quad i=1, \ldots, m$,

for a certain symmetric $H$. Consider the subspace $V=V_{i}^{*} \cap \hat{V}_{i}^{*}$ for an $i \in\{1, \ldots, m\}$. Clearly $V \subset$ Ker $C$, so $(A+B H C) V=A V \subset V$ and thus by the observability of the system $V=0$. Hence for $i=$ 
$1, \ldots, m$,

$V_{i}^{*} \cap \sum_{j \neq i} V_{j}^{*}=0$

Moreover by controllability we have $\sum_{i=1}^{m} V_{i}^{*}=\mathbb{R}^{2 n}$. We will now use Proposition 2.1 for showing that condition (b) is satisfied. Since

$(A+B H C) V_{i}^{*} \subset V_{i}^{*}$

the subspace $V_{i}^{*}$ is $(C, A)$-invariant and so $\left(V_{i}^{*}\right)^{\perp}$ is $(A, B)$-invariant. Because

$\left(V_{i}^{*}\right)^{\perp} \subset\left(\operatorname{Im} b_{i}\right)^{\perp}=\operatorname{Ker} \hat{C}_{i}$

we obtain

$\left(V_{i}^{*}\right)^{\perp} \subset \hat{V}_{i}^{*}, \quad i=1, \ldots, m$.

However since

$\operatorname{dim}\left(V_{i}^{*}\right)^{\perp}=2 n-\operatorname{dim} V_{i}^{*}$

and

$\mathbb{R}^{2 n}=V_{i}^{*} \oplus \hat{V}_{i}^{*}, \quad i=1, \ldots, m$,

we may conclude $\left(V_{i}^{*}\right)^{\perp}=\hat{V}_{i}^{*}$ for all $i=1, \ldots, m$.

Remark. Notice that if (a) and (b) are satisfied $V_{i}^{*}$ is not only the maximal $(A, B)$-invariant subspace contained in $\operatorname{Ker} \hat{C}_{i}$, but also equal to the minimal $(C, A)$-invariant subspace containing $\operatorname{Im} b_{i}, i=$ $1, \ldots, m$. Furthermore it follows that $V_{i}^{*}$ equals $R^{*}\left(A, B, \hat{C}_{i}\right)$.

We conclude from the above theorem that the Hamiltonian input-output decoupling problem is solvable if and only if the Hamiltonian system $\dot{x}=A x+B u, y=C x$ with $V^{*}(A, B, C)=0$ can be partitioned into $m$ independent Hamiltonian subsystems after applying Hamiltonian feedback. Furthermore the diagonal elements of the output feedback $u=H y$ remain to be freely chosen. This freedom can be used to adjust the behaviour of these subsystems. We leave the case $V^{*}(A, B, C)$ $\neq 0$ for further research (see also [11]).

In a similar way as in Theorem 3.1 we can deal with the Hamiltonian block-decoupling problem, that there is given a partitioning of the outputs

$y=\left(y_{1}, \ldots, y_{m}\right)^{\mathbf{T}}=\left(\underline{y}_{1}, \ldots, \underline{y}_{k}\right)^{\mathrm{T}}$ into $k$ disjoint groups (and thus also of the inputs $\left.u=\left(u_{1}, \ldots, u_{m}\right)^{\mathrm{T}}=\left(\underline{u}_{1}, \ldots, \underline{u}_{k}\right)^{\mathrm{T}}\right)$

and the question is whether or not we can find a Hamiltonian feedback $u=H x+v, H=H^{\mathrm{T}}$, such that $\underline{v}_{i}$ does not affect $\underline{y}_{j}, j \neq i$, and $\underline{v}_{i}$ controls $\underline{y}_{i}$, $i=1, \ldots, k$. Writing, as before, $B_{i}$ for the input matrix corresponding to the inputs $\underline{u}_{i}, \hat{B}_{i}$ for the input matrix $B$ with deleting $B_{i}$ and similarly $C_{i}$ and $\hat{C}_{i}$ we obtain the following sufficient conditions.

Corollary 3.2. Let $(A, B, C)$ be a minimal Hamiltonian system with $V^{*}(A, B, C)=0$. Then the Hamiltonian block-decoupling problem is solvable if the following three conditions hold:

(a) $\operatorname{Im} B_{i} \in V^{*}\left(A, B, \hat{C}_{i}\right), i=1, \ldots, k$,

(b) $V^{*}\left(A, B, C_{i}\right)=\left(V^{*}\left(A, B, \hat{C}_{i}\right)\right)^{\perp}, \quad i=$ $1, \ldots, k$,

(c) $R^{*}\left(A, B_{i}, C_{i}\right)=0, i=1, \ldots, k$.

Proof. Let

$V_{i}^{*}:=V^{*}\left(A, B, C_{i}^{*}\right)$ and $\hat{V}_{i}^{*}=V^{*}\left(A, B, C_{i}\right)$,

$i=1, \ldots, k$. Since $\operatorname{Im} B_{i} \in V_{i}^{*}$ we have $V_{i}^{*}=$ $V^{*}\left(A, \hat{B}_{i}, \hat{C}_{i}\right)$ and because $\hat{V}_{i}^{*}=\sum_{j{ }_{i}} V_{j}^{*}$ we obtain $\hat{V}_{i}^{*}=V^{*}\left(A, B_{i}, C_{i}\right)$. The triple $\left(A, B_{i}, C_{i}\right)$ being Hamiltonian allows us to apply Proposition 2.1 to conclude

$0=R^{*}\left(A, B_{i}, C_{i}\right)=\hat{V}_{i}^{*} \cap\left(\hat{V}_{i}^{*}\right)^{\perp}$,

$j=1, \ldots, k$. So again, the $\hat{V}_{i}^{*}$ 's are symplectic subspaces and

$0=\hat{V}_{i}^{*} \cap\left(\hat{V}_{i}^{*}\right)^{\perp}=\sum_{j \neq i} V_{j}^{*} \cap V_{i}^{*}$

implies that they are independent. The rest of the proof is now as in Theorem 3.1.

Remark. Condition (c) of Corollary 3.2 implies that the subsystems $\left(A, B, C_{i}\right)$ are left-invertible, cf. [10], and therefore also right-invertible. In this way the 'geometric' decoupling problem with the prerequisite of output controllability coincides with the transfer function approach of [3].

We will conclude this paper with a very simple example. 
Example 3.3. Time-reversible Hamiltonian systems have the following additional structure (cf. [6]). There exists a symplectic basis of $\mathbb{R}^{2 n}$ such that

$A=\left(\begin{array}{cc}0 & P \\ -Q & P\end{array}\right), \quad B=\left(\begin{array}{l}0 \\ B_{1}\end{array}\right), \quad C=\left(\begin{array}{ll}C_{1} & 0\end{array}\right)$,

with $P=P^{\mathrm{T}}, Q=Q^{\mathrm{T}}$ and $B_{1}^{\mathrm{T}}=C_{1}$. Considering only the matrix $A$ we know that if $P>0$ there is a symplectic basis for $\mathbb{R}^{2 n}$ such that

$A=\left(\begin{array}{cc}0 & I \\ -D & 0\end{array}\right)$

with $D$ a diagonal matrix. Setting $B_{1}=I_{m}=C_{1}$ $(m=n)$, we clearly have a decoupled system consisting of $n$ independent oscillators. However, given $B_{1}=C_{1}=I_{n}$, input-output decoupling is possible if and only if $P$ is already diagonal. $(Q$ may be arbitrary since $Q+I_{n} H I_{n}$ can be freely assigned by the choice of $H$.)

\section{References}

[1] R.W. Brockett and A. Rahimi, Lie algebras and linear differential equations, in L. Weiss, Ed., Ordinary Differential Equations (Academic Press, New York, 1972).
[2] P.L. Falb and W.A. Wolovich, Decoupling in the design of multivariable control systems, IEEE Trans. Automat. Control 12 (1967) 651-659.

[3] M.L.J. Hautus and M. Heymann, Linear feedback decoupling - transfer function analysis, IEEE Trans. Automat. Control 28 (1983) 823-832.

[4] A.S. Morse, Structural invariants of linear multivariable systems, SIAM J. Control 11 (1973) 446-465.

[5] A.S. Morse and W.M. Wonham, Status of noninteracting control, IEEE Trans. Automat. Control 16 (1971) 568-580

[6] A.J. van der Schaft, Time-reversible Hamiltonian systems, Systems Control Lett. 1 (1982) 295-300.

[7] A.J. van der Schaft, Disturbance decoupling by observation feedback for Hamiltonian systems, Systems Control Lett. 2 (1983) 286-291.

[8] A.J. van der Schaft, System theoretic descriptions of physical systems, Doctoral Dissertation, Rijksuniversiteit Groningen (1983); also CWI Tracts, No. 3, Amsterdam (1984).

[9] J.M. Schumacher, Dynamic feedback in finite- and infinite-dimensional linear systems, Mathematical Centre Tract 143 (1981).

[10] W.M. Wonham, Linear Multivariable Control: A Geometric Approach (Springer, Berlin, 1979).

[11] H. Nijmeijer and A.J. van der Schaft, Input-output decoupling of Hamiltonian systems: The nonlinear case, in preparation. 